\title{
Repeat convection-enhanced delivery for diffuse intrinsic pontine glioma
}

\author{
*Evan D. Bander, MD, ${ }^{1,2}$ Alexander D. Ramos, MD, PhD, ${ }^{1,2}$ Eva Wembacher-Schroeder, BS, ${ }^{3}$ \\ Iryna Ivasyk, BS, ${ }^{1}$ Rowena Thomson, PhD, ${ }^{3}$ Peter F. Morgenstern, MD, ${ }^{4,5}$ \\ and Mark M. Souweidane, MD1,2
}

${ }^{1}$ Department of Neurological Surgery, NewYork-Presbyterian Hospital, Weill Cornell Medicine, New York; ${ }^{2}$ Department of Neurosurgery, Memorial Sloan Kettering Cancer Center, New York, New York; ${ }^{3}$ Brainlab AG, Munich, Germany; and Departments of ${ }^{4}$ Neurosurgery and ${ }^{5}$ Pediatrics, Icahn School of Medicine at Mount Sinai, New York, New York

\begin{abstract}
OBJECTIVE While the safety and efficacy of convection-enhanced delivery (CED) have been studied in patients receiving single-dose drug infusions, agents for oncological therapy may require repeated or chronic infusions to maintain therapeutic drug concentrations. Repeat and chronic CED infusions have rarely been described for oncological purposes. Currently available CED devices are not approved for extended indwelling use, and the only potential at this time is for sequential treatments through multiple procedures. The authors report on the safety and experience in a group of pediatric patients who received sequential CED into the brainstem for the treatment of diffuse intrinsic pontine glioma.
\end{abstract}

METHODS Patients in this study were enrolled in a phase I single-center clinical trial using ${ }^{124} \mid-8 \mathrm{H} 9$ monoclonal antibody (124-omburtamab) administered by CED (clinicaltrials.gov identifier NCT01502917). A retrospective chart and imaging review were used to assess demographic data, CED infusion data, and postoperative neurological and surgical outcomes. MRI scans were analyzed using iPlan Flow software for volumetric measurements. Target and catheter coordinates as well as radial, depth, and absolute error in MRI space were calculated with the ClearPoint imaging software.

RESULTS Seven patients underwent 2 or more sequential CED infusions. No patients experienced Clinical Terminology Criteria for Adverse Events grade 3 or greater deficits. One patient had a persistent grade 2 cranial nerve deficit after a second infusion. No patient experienced hemorrhage or stroke postoperatively. There was a statistically significant decrease in radial error $(p=0.005)$ and absolute tip error $(p=0.008)$ for the second infusion compared with the initial infusion. Sequential infusions did not result in significantly different distribution capacities between the first and second infusions (volume of distribution determined by the PET signal/volume of infusion ratio [mean \pm SD]: $2.66 \pm 0.35$ vs 2.42 $\pm 0.75 ; p=0.45$ ).

CONCLUSIONS This series demonstrates the ability to safely perform sequential CED infusions into the pediatric brainstem. Past treatments did not negatively influence the procedural workflow, technical application of the targeting interface, or distribution capacity. This limited experience provides a foundation for using repeat CED for oncological purposes.

https://thejns.org/doi/abs/10.3171/2020.6.PEDS20280

KEYWORDS convection-enhanced delivery; repeat infusion; diffuse intrinsic pontine glioma; distribution; oncology

A $\mathrm{N}$ expanding repertoire of platforms, including brachytherapy, drug-impregnated biodegradable wafers, osmotic or ultrasonic interruption of the blood-brain barrier (BBB), and convection-enhanced delivery (CED), exist to enhance drug delivery into the brain. ${ }^{1}$ CED utilizes positive pressure to create a convective flow of molecules capable of achieving a greater overall drug distribution compared with simple diffusion. ${ }^{2-4}$ The safety and efficacy of CED for oncological purposes has primarily been studied in the preclinical setting and in patients receiving single-dose drug infusions..$^{5-10}$ Logically, antineoplastic agents would benefit from repeated or chronic infusions to maintain therapeutic concentrations and sustainable long-term results. ${ }^{11,12}$ Repeat or chronic infusions

ABBREVIATIONS BBB = blood-brain barrier; $C E D=$ convection-enhanced delivery; CTCAE = Clinical Terminology Criteria for Adverse Events version 4.0; DIPG = diffuse intrinsic pontine glioma; $\mathrm{Vd}=$ volume of distribution determined by the PET signal; $\mathrm{Vi}=$ volume of infusion.

SUBMITTED April 13, 2020. ACCEPTED June 1, 2020.

INCLUDE WHEN CITING Published online September 25, 2020; DOI: 10.3171/2020.6.PEDS20280.

${ }^{*}$ E.D.B. and A.D.R. contributed equally to this work. 
have been demonstrated in large animal models in the supratentorial compartment. ${ }^{13,14}$ Platforms for continued or repeated infusions are under development. FDA-approved infusion cannulas and catheters are not cleared for extended (> 72 hours) indwelling purposes. At this time, repeated infusions are limited to the use of sequential procedures for cannula/catheter insertion. We reviewed a subgroup of children who were treated with sequential therapeutic drug administration by CED into the brainstem for the treatment of diffuse intrinsic pontine glioma (DIPG).

\section{Methods}

\section{Patient Selection}

Patients in this study were enrolled in a phase I singlecenter clinical trial using ${ }^{124} \mathrm{I}-8 \mathrm{H} 9$ monoclonal antibody ( ${ }^{124}$ I-omburtamab, Y-mAbs Therapeutics) administered by CED. All patients were radiographically diagnosed with DIPG and were previously treated with external-beam radiation therapy prior to CED (clinicaltrials.gov identifier NCT01502917). An option for CED retreatment at the same dose level was offered if patients exhibited no doselimiting toxicity or disease progression beyond the 30-day observation window. Informed consent was obtained from all patients or their parents/legal guardians. Between 2012 and 2019, 7 patients in this trial were treated with more than one CED infusion. Retrospective chart and imaging review were used to assess demographic data, CED infusion data, and postoperative surgical complications.

\section{Surgical Technique/Outcomes}

Repeat CED was performed using a supratentorial trajectory with intraprocedural MRI-guided stereotactic placement (ClearPoint, MRI Interventions Inc.) of a small-caliber infusion catheter (Brainlab Flexible Catheter, Brainlab), as previously described. ${ }^{6}$ Infusion volumes and dose were prescribed according to enrollment in the clinical trial. Unique entry sites were chosen, and the previous twist-drill site was not used. Trajectories for repeat infusions were chosen based on anatomical constraints, with the goal of optimizing distribution and avoiding conflict with previous catheter paths when possible. Radial error, depth error, target, and catheter coordinates in MRI space were obtained using the ClearPoint imaging software, and absolute tip error was calculated, as previously described..$^{15}$

An evaluation period of 30 days was used to report on treatment-related toxicity. Toxicity was assessed and graded according to the Clinical Terminology Criteria for Adverse Events version 4.0 (CTCAE). Adverse events were assessed multiple times per day while the patient was hospitalized and at weekly outpatient visits thereafter. Adverse events were considered persistent or permanent if present after 30 days of follow-up.

\section{MRI and Analysis}

For the imaging analysis, volumetric 3D T1-weighted gradient echo brain MRI (slice thickness $1 \mathrm{~mm}$, matrix $256 \times 256$, slice spacing $0.5 \mathrm{~mm}$, FOV $22 \mathrm{~cm}$ ) was performed within 14 days prior to catheter placement, as well as on postoperative day 1 after drug infusion. Data were acquired using a 3-T GE Signa scanner (GE Healthcare). PET/CT images of the brain performed within 1-6 hours after completion of drug infusion using a GE PET/CT scanner and MRI scans were imported into iPlan Flow software (Brainlab) and merged for analysis.

\section{Trajectory Definition}

The actual catheter position could be identified as a signal void on MRI. A virtual trajectory was added for the catheter depicted on MR images of the initial and repeat infusion procedure. The probe view functionality in iPlan Flow displays image slices from the perspective of a trajectory angle. Scrolling along the course of a trajectory allows for determination of intersections between the actual catheter and the previous catheter track. The distance from the cross-section to the catheter target point is displayed in the software (Fig. 1).

\section{Evaluation of Drug Distribution}

The volume of infusate distribution (Vd) was analyzed on PET/CT imaging using a threshold-based segmentation method. This method was previously assessed for the best volumetric and geometrical match between PET and distribution volume determined based on differences in T2-weighted images acquired pre- and postinfusion in patients out of the same NCT01502917 study, with only minimal preexisting $\mathrm{T} 2$ signal abnormality at the injection site (unpublished work). A PET threshold of $27 \%$ allowed for generation of objects with high similarity to the reference objects and therefore was used within this study for automatic segmentation of the drug distribution. For each catheter, the volume of infusion (Vi) was used to calculate the $\mathrm{Vd} / \mathrm{Vi}$ ratio.

\section{Statistical Analysis}

Unpaired two-sample t-tests were used to compare quantitative variables between two infusions; $p<0.05$ was considered statistically significant. Microsoft Excel (Microsoft Corp.) was used for statistical analysis.

\section{Results}

\section{Demographics and Clinical Outcomes}

Seven patients ( 3 female and 4 male) underwent 2 or more CED infusions (Table 1). Six patients had 2 infusions, while 1 patient underwent 3 infusions. The mean patient age was 5.4 years at diagnosis. The mean time between infusions was $102 \pm 70$ days (range 50-268 days). The mean infusion volumes $( \pm \mathrm{SD})$ were similar among the 3 infusions $(3.20 \pm 1.3$ vs $3.15 \pm 1.3$ vs $4.14 \mathrm{~mL}$; Table 2). A trajectory through the left hemisphere was chosen for $71 \%$ of patients $(n=5)$. All patients underwent a second infusion through an ipsilateral approach $(n=7)$. One patient had 3 infusions, of which the last infusion was approached from the contralateral side.

No patients in this cohort experienced CTCAE grade 3 or greater adverse events. Adverse events were limited to grade 1 and 2 events, which included contralateral hemiparesis, nystagmus, dysmetria, and/or cranial nerve VI and VII palsies. All but one of these neurological adverse 

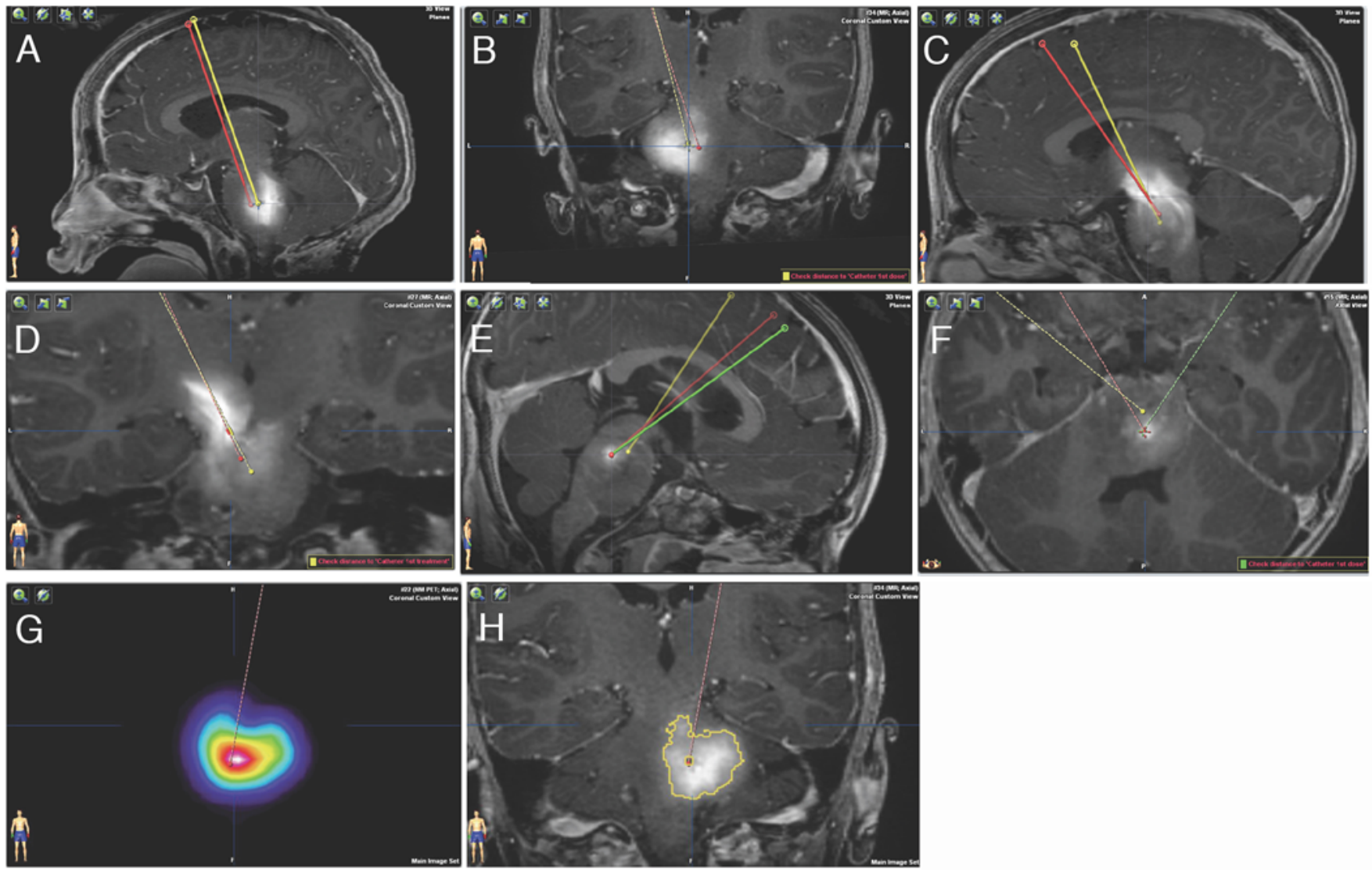

FIG. 1. Representative iPlan Flow MR images comparing sequential (red = infusion 1; yellow = infusion 2; green = infusion 3) trajectories for 3 patients ( $A$ and $B, C$ and $D$, and $E$ and $F$ ) in 2 planes. A and B: Appropriate tumor coverage of gadolinium coinfusion can be visualized. $\mathbf{C}$ and D: Leakage of gadolinium coinfusion along a prior cannula track. $\mathbf{E}$ and $\mathrm{F}$ : Gadolinium coinfusion from the third infusion can be seen partially pooling in a previous infusion site. $\mathbf{G}$ and $\mathbf{H}$ : Representative MR images obtained in a fourth patient after a repeat drug infusion, demonstrating T1 gadolinium coinfusion $(G)$ and PET $(H)$ signal postinfusion with good shape concordance. Figure is available in color online only.

events resolved, with the only persistent deficit being a partial facial paresis and mild abducens palsy. No patients experienced hemorrhage or stroke postoperatively.

\section{Targeting Accuracy}

There was no intraprocedural need for repositioning of the MR-compatible frame or infusion catheter. Table 2 demonstrates the radial error, depth error, and absolute tip error, derived from comparing the intended target with the actual catheter tip location seen during intraprocedural MRI. ${ }^{15}$ Data were unavailable for infusion 1 for one patient. There was a statistically significant decrease in radial error $(p=0.005)$ and absolute tip error $(p=0.008)$ in the second infusion compared with the initial infusion. There was no difference in depth error $(p=0.64)$.

\section{Distribution Volume}

Using the theranostic capacity of the PET-labeled antibody, a standardized threshold was chosen and applied across all patients and infusions to determine the drug distribution volume and then normalized based on infusion volume to determine the $\mathrm{Vd} / \mathrm{Vi}$ ratio. Sequential infusions did not result in significantly different distribution capaci- ties between the first and second infusions ( $\mathrm{Vd} / \mathrm{Vi}$ ratio: $2.66 \pm 0.35$ vs $2.42 \pm 0.75 ; p=0.45$ ). One patient had leakage of infusate into a previous catheter track. Two patients had pooling of infusate into a previous injection site. All 3 of these cases occurred in patients whose sequential catheter trajectories crossed within $15 \mathrm{~mm}$ of the catheter tip. In the 2 cases in which pooling of infusate was found, it is notable that the $\mathrm{Vd} / \mathrm{Vi}$ ratio was lower compared with the initial infusions (Table 1).

\section{Discussion}

CED offers the ability to bypass the BBB and achieve efficient drug delivery to the CNS. The convective flow of CED produces drug distribution volumes exceeding that of simple diffusion and also allows for significant concentration falloff at the borders, limiting toxicity to surrounding tissue. ${ }^{2}$ These factors are optimal for oncological treatment, as many antineoplastic agents are poorly permeable through the $\mathrm{BBB}$, and doses required to achieve tumor efficacy come at the cost of systemic toxicity. ${ }^{16}$ A number of preclinical and clinical trials have demonstrated the safety of single-dose CED. . $^{2,6-8,10,17-19}$ However, in the setting of malignant brain tumors, a single-dose treatment is likely 
TABLE 1. Summary of demographics and clinical outcomes after sequential infusions

\begin{tabular}{|c|c|c|c|c|c|c|c|}
\hline $\begin{array}{l}\text { Patient } \\
\text { No. }\end{array}$ & Sex & Event & $\begin{array}{l}\text { Age } \\
\text { (yrs) }\end{array}$ & Laterality & Postinfusion Deficits* & Vd/Vi Ratio & $\begin{array}{l}\text { Infusate Pooling } \\
\text { or Leakage }\end{array}$ \\
\hline \multirow{3}{*}{1} & \multirow{3}{*}{$\mathrm{F}$} & Diagnosis & 5.43 & & & & \\
\hline & & Infusion 1 & 5.77 & Lt & Nystagmus & 2.8 & \\
\hline & & Infusion 2 & 6.05 & $\mathrm{Lt}$ & & 1.77 & Neither \\
\hline \multirow{3}{*}{2} & \multirow{3}{*}{$\mathrm{F}$} & Diagnosis & 5.72 & & & & \\
\hline & & Infusion 1 & 6.06 & $\mathrm{Rt}$ & & 2.41 & \\
\hline & & Infusion 2 & 6.31 & Rt & IL CN VI, CN VII (grade 1), ataxia & 2.30 & Pooling \\
\hline \multirow{3}{*}{3} & \multirow{3}{*}{$\mathrm{F}$} & Diagnosis & 7.67 & & & & \\
\hline & & Infusion 1 & 8.11 & $\mathrm{Lt}$ & & 3.31 & \\
\hline & & Infusion 2 & 8.30 & $\mathrm{Lt}$ & & 3.36 & Leakage \\
\hline \multirow{3}{*}{4} & \multirow{3}{*}{ M } & Diagnosis & 2.88 & & & & \\
\hline & & Infusion 1 & 3.25 & Lt & & 2.20 & \\
\hline & & Infusion 2 & 3.99 & $\mathrm{Lt}$ & & 3.10 & Neither \\
\hline \multirow{3}{*}{5} & \multirow{3}{*}{ M } & Diagnosis & 3.99 & & & & \\
\hline & & Infusion 1 & 4.30 & Rt & CL hemiparesis (grade 1) & 2.58 & \\
\hline & & Infusion 2 & 4.48 & $\mathrm{Rt}$ & CL hemiparesis (grade 2) & & \\
\hline \multirow{3}{*}{6} & \multirow{3}{*}{ M } & Diagnosis & 6.86 & & & & \\
\hline & & Infusion 1 & 7.29 & Lt & IL CN VI, CN VII (grade 1), dysmetria, nystagmus & 2.58 & \\
\hline & & Infusion 2 & 7.60 & $\mathrm{Lt}$ & CL hemiparesis (grade 1); IL CN VI, VII (grade 2) & 1.41 & Neither \\
\hline \multirow{4}{*}{7} & \multirow{4}{*}{ M } & Diagnosis & 5.39 & & & & \\
\hline & & Infusion 1 & 5.73 & Lt & CL hemiparesis (grade 2) & 2.76 & \\
\hline & & Infusion 2 & 5.87 & Lt & CL hemiparesis (grade 1) & 2.56 & Neither \\
\hline & & Infusion 3 & 6.04 & Rt & IL hemiparesis (grade 1), CN VII; CL CN VI & 1.86 & Pooling \\
\hline
\end{tabular}

$\mathrm{CL}=$ contralateral $\mathrm{CN}=$ cranial nerve; $\mathrm{IL}=$ ipsilateral .

* Grades are CTCAE grades.

insufficient to target and achieve toxicity in all neoplastic cells, particularly those that are beyond the distribution volume of a single target or in resistant stages of the cell cycle. ${ }^{11}$ Repeat CED infusion has been performed in limited cases for malignant glioma and DIPG..$^{5,20-22}$ However, there is a need for further foundational research on the

TABLE 2. CED targeting and infusion outcomes by procedure

\begin{tabular}{lcccc}
\hline & Infusion 1 & Infusion 2 & Infusion 3 & p Value* \\
\hline No. of patients & 7 & 7 & 1 & \\
\hline Age (yrs) & 5.79 & 6.09 & 6.04 & \\
\hline Hemisphere (It/rt) & $5 / 2$ & $5 / 2$ & $0 / 1$ & \\
\hline $\begin{array}{l}\text { Mean infusion vol } \\
\text { (mL) }\end{array}$ & $3.20 \pm 1.30$ & $3.15 \pm 1.30$ & 4.14 & 0.94 \\
\hline Radial error (mm) & $2.93 \pm 1.24 \dagger$ & $1.07 \pm 0.64$ & 0.30 & 0.005 \\
\hline Depth error (mm) & $0.38 \pm 0.24 \dagger$ & $0.48 \pm 0.47$ & 0.10 & 0.64 \\
\hline $\begin{array}{l}\text { Absolute tip error } \\
\text { (mm) }\end{array}$ & $2.97 \pm 1.23 \dagger$ & $1.27 \pm 0.61$ & 0.37 & 0.008 \\
\hline Vd/Vi & $2.66 \pm 0.35$ & $2.42 \pm 0.75 \dagger$ & 1.86 & 0.45 \\
\hline
\end{tabular}

Values represent the number of patients (\%) unless stated otherwise. Mean values are presented as the mean $\pm \mathrm{SD}$.

${ }^{*}$ Calculated between infusions 1 and 2 .

† Six patients had data available for calculations. use of repeated or chronic infusions in neuro-oncology, particularly for DIPG, due to distinct drug toxicity profiles within the brainstem. ${ }^{23}$ This report begins to address that perceived need. In this series, 6 patients underwent 2 infusions and 1 patient underwent 3 infusions. Past treatments did not negatively influence the procedural work flow, technical application of the targeting interface, or drug distribution capacity. The repeated infusions resulted in no CTCAE grade 3 or greater adverse events. While limited, the safety and technical feasibility of our experience support the capability of sequential treatments as an acceptable management approach.

The use of repeated targeting procedures and infusion in this cohort exemplifies the current limitations of existing devices and regulations. Infusion catheters designed for CED, in general, are approved only for short-term use (i.e., < 72 hours). Off-label use of previously approved long-term lumbar catheters and infusion pumps has been described and implemented in animal models and is currently in a clinical trial for malignant glioma in adults (clinicaltrials.gov identifier NCT03154996). ${ }^{14}$ In addition, novel long-term CED infusion devices are under study in preclinical models as well as a clinical trial for Parkinson's disease (NCT03775538). ${ }^{13,14}$ However, to date, no FDA-approved long-term infusion system designed specifically for CED is available for use. The FDA considers infusion devices as a combination product with the drug 
being infused, and since all current drugs are still experimental in various clinical trial phases, this results in a difficult approval process. There is a clear need for more comprehensive exploration in device design and regulatory approval for interfaces that avoid repeated surgery for cannula/catheter deployment. However, in the interim, it is essential to assess and report on the safety and feasibility of repeat procedures, as in this series.

From a technical perspective, repeat CED demonstrated no decrease in targeting accuracy or drug distribution. The mean $\mathrm{Vd} / \mathrm{Vi}$ ratios in this cohort fall within the range (1.1-4) of that in the existing literature, as measured by a variety of methods, including SPECT, gadolinium coinfusion, or T2-weighted MRI, and were not significantly degraded in the serial infusions. , $^{5,8,10,24}$ There was an intent to optimize distribution volume in preoperative trajectory planning. Based on our experience, catheters that crossed farther from the cannula tip did not result in leakage or backflow of infusate. Furthermore, targets that avoid previous infusion cavities can minimize the risk of infusate pooling. These factors should be taken into consideration for future planning. From a safety perspective, similar to the wider clinical trial, there were no CTCAE grade 4 or greater events..$^{10}$ Given the early onset and transient nature of the grade 1 and 2 events, these were likely due to transient inflammation from catheter placement or from transient deformation related to the infusion and are unlikely to be related to any toxicity from the drug.

This case series is limited by its small sample size and small range of infusion volumes. Distribution volumes were determined by using a common threshold for PET signal across all patients and infusions to maintain a standardized method for all measurements. However, the reliability and accuracy of the PET signal for the distribution volume compared with coinfusion of gadolinium or measuring the T2-weighted MR signal remains unclear. Due to the preexisting T2 signal in the brainstem after the initial infusions, T2 distribution volumes were not considered valid in the repeat infusion cases. ${ }^{24,25}$ Furthermore, gadolinium coinfusion was only performed on repeat infusion and, therefore, did not allow for comparison between sequential infusions. The theranostic capacity of the infused drug allowed for a standardized method to assess distribution across all patients and infusions. It comes with the caveat, however, that the PET signal threshold used was optimized based on a separate subset of data that may not have equivalent accuracy to all infusions. In this cohort of patients, there was notably good concordance of shape between the PET and gadolinium signals for all infusions in which they were both employed. However, depending on the thresholds applied for volumetric measurement, there was some variation in the gadolinium- and PET-calculated distribution volumes. A more precise paradigm for comparison of infusate distribution between the first and subsequent infusions and between measurement methods is needed and will be a topic of future studies. Furthermore, work is needed in the design and approval of long-term CED interfaces, as well as continued assessment of safety and best practices of repeat or chronic CED infusions.

\section{Conclusions}

This series demonstrates the ability to safely perform repeated CED infusions into the pediatric brainstem. Past treatments did not negatively influence the procedural workflow, technical application of the targeting interface, accuracy of catheter placement, or distribution capacity. The findings in this study should be taken into consideration during the planning process for future sequential CED cases. This limited experience provides a foundation for using repeat CED in the brainstem for children with DIPG.

\section{Acknowledgments}

We thank the patients and their families who participated in the trial. We also thank the following organizations and programs for research support: The Cure Starts Now; Solving Kids' Cancer; Lyla Nsouli Foundation for Children's Brain Cancer Research; Cookies for Kids' Cancer; Cristian Rivera Foundation; Battle for a Cure; Cole Foundation; Meryl and Charles Witmer Charitable Foundation; Tuesdays with Mitch Charitable Foundation; Ian's Friends Foundation; The Matthew Larson Foundation for Pediatric Brain Tumors; McKenna Claire Foundation; The Olivia Boccuzzi Foundation; Children's Brain Tumor Family Foundation; Pediatric Brain Tumor Foundation; St. Baldrick's Foundation; Lyonhearted Foundation; The ChadTough Foundation; Fly A Kite Foundation; and The Brooke Healey Foundation.

Ms. Ivasyk was supported by a Medical Scientist Training Program grant from the National Institute of General Medical Sciences of the National Institutes of Health (NIH) under award no. T32GM007739 to the Weill Cornell, Rockefeller, Sloan Kettering Tri-Institutional MD-PhD Program. This study is solely the responsibility of the authors and does not necessarily represent the official views of the NIH.

\section{References}

1. Hendricks BK, Cohen-Gadol AA, Miller JC. Novel delivery methods bypassing the blood-brain and blood-tumor barriers. Neurosurg Focus. 2015;38(3):E10.

2. Bobo RH, Laske DW, Akbasak A, et al. Convection-enhanced delivery of macromolecules in the brain. Proc Natl Acad Sci U S A. 1994;91(6):2076-2080.

3. Lonser RR, Walbridge S, Garmestani K, et al. Successful and safe perfusion of the primate brainstem: in vivo magnetic resonance imaging of macromolecular distribution during infusion. J Neurosurg. 2002;97(4):905-913.

4. Lonser RR, Sarntinoranont M, Morrison PF, Oldfield EH. Convection-enhanced delivery to the central nervous system. J Neurosurg. 2015;122(3):697-706.

5. Barua NU, Lowis SP, Woolley M, et al. Robot-guided convection-enhanced delivery of carboplatin for advanced brainstem glioma. Acta Neurochir (Wien). 2013;155(8): 1459-1465.

6. Kunwar S, Chang S, Westphal M, et al. Phase III randomized trial of CED of IL13-PE38QQR vs Gliadel wafers for recurrent glioblastoma. Neuro Oncol. 2010;12(8):871-881.

7. Lonser RR, Schiffman R, Robison RA, et al. Image-guided, direct convective delivery of glucocerebrosidase for neuronopathic Gaucher disease. Neurology. 2007;68(4):254-261.

8. Sampson JH, Brady ML, Petry NA, et al. Intracerebral infusate distribution by convection-enhanced delivery in humans with malignant gliomas: descriptive effects of target anatomy and catheter positioning. Neurosurgery. 2007;60(2) (suppl 1):ONS89-ONS99.

9. Sampson JH, Brady M, Raghavan R, et al. Colocalization of gadolinium-diethylene triamine pentaacetic acid with high- 
molecular-weight molecules after intracerebral convectionenhanced delivery in humans. Neurosurgery. 2011;69(3): $668-676$.

10. Souweidane MM, Kramer K, Pandit-Taskar N, et al. Convection-enhanced delivery for diffuse intrinsic pontine glioma: a single-centre, dose-escalation, phase 1 trial. Lancet Oncol. 2018;19(8):1040-1050.

11. Butowski NA, Bringas JR, Bankiewicz KS, Aghi MK. Editorial. Chronic convection-enhanced delivery: the next frontier in regional drug infusion for glioblastoma. J Neurosurg. 2020;133(3):611-613.

12. Lewis O, Woolley M, Johnson D, et al. Chronic, intermittent convection-enhanced delivery devices. J Neurosci Methods. 2016;259:47-56.

13. Barua NU, Woolley M, Bienemann AS, et al. Intermittent convection-enhanced delivery to the brain through a novel transcutaneous bone-anchored port. J Neurosci Methods. 2013;214(2):223-232.

14. D'Amico RS, Neira JA, Yun J, et al. Validation of an effective implantable pump-infusion system for chronic convectionenhanced delivery of intracerebral topotecan in a large animal model. J Neurosurg. 2020;133(3):614-623.

15. Mohyeldin A, Lonser RR, Elder JB. Real-time magnetic resonance imaging-guided frameless stereotactic brain biopsy: technical note. J Neurosurg. 2016;124(4):1039-1046.

16. Zhou Z, Singh R, Souweidane MM. Convection-enhanced delivery for diffuse intrinsic pontine glioma treatment. Curr Neuropharmacol. 2017;15(1):116-128.

17. Chen MY, Lonser RR, Morrison PF, et al. Variables affecting convection-enhanced delivery to the striatum: a systematic examination of rate of infusion, cannula size, infusate concentration, and tissue-cannula sealing time. J Neurosurg. 1999;90(2):315-320.

18. Luther N, Zhou Z, Zanzonico P, et al. The potential of theragnostic ${ }^{124} \mathrm{I}-8 \mathrm{H} 9$ convection-enhanced delivery in diffuse intrinsic pontine glioma. Neuro Oncol. 2014;16(6):800-806.

19. Jagannathan J, Walbridge S, Butman JA, et al. Effect of ependymal and pial surfaces on convection-enhanced delivery. $J$ Neurosurg. 2008;109(3):547-552.

20. Heiss JD, Jamshidi A, Shah S, et al. Phase I trial of convection-enhanced delivery of IL13-Pseudomonas toxin in children with diffuse intrinsic pontine glioma. J Neurosurg Pediatr. 2018;23(3):333-342.

21. Patel SJ, Shapiro WR, Laske DW, et al. Safety and feasibility of convection-enhanced delivery of Cotara for the treatment of malignant glioma: initial experience in 51 patients. Neurosurgery. 2005;56(6):1243-1253.

22. Chittiboina P, Heiss JD, Warren KE, Lonser RR. Magnetic resonance imaging properties of convective delivery in diffuse intrinsic pontine gliomas. J Neurosurg Pediatr. 2014; 13(3):276-282.
23. Sewing ACP, Lagerweij T, van Vuurden DG, et al. Preclinical evaluation of convection-enhanced delivery of liposomal doxorubicin to treat pediatric diffuse intrinsic pontine glioma and thalamic high-grade glioma. J Neurosurg Pediatr. 2017; 19(5):518-530.

24. Sampson JH, Raghavan R, Provenzale JM, et al. Induction of hyperintense signal on T2-weighted MR images correlates with infusion distribution from intracerebral convectionenhanced delivery of a tumor-targeted cytotoxin. AJR Am J Roentgenol. 2007;188(3):703-709.

25. Richardson RM, Gimenez F, Salegio EA, et al. T2 imaging in monitoring of intraparenchymal real-time convectionenhanced delivery. Neurosurgery. 2011;69(1):154-163.

\section{Disclosures}

Memorial Sloan Kettering Cancer Center has institutional financial interests related to this research in the form of intellectual property rights and equity interests in Y-mAbs, the company licensing the intellectual property from Memorial Sloan Kettering Cancer Center. Omburtamab was licensed to Y-mAbs Therapeutics in 2015. This clinical trial was sponsored by Y-mAbs Therapeutics since 2017. Ms. Wembacher-Schroeder and Dr. Thomson are employees of Brainlab.

\section{Author Contributions}

Conception and design: Souweidane, Bander, Ramos, Wembacher-Schroeder. Acquisition of data: Souweidane, Bander, Ramos, Wembacher-Schroeder, Ivasyk, Morgenstern. Analysis and interpretation of data: Souweidane, Bander, Ramos, Wembacher-Schroeder, Ivasyk, Morgenstern. Drafting the article: Souweidane, Bander, Ramos, Thomson. Critically revising the article: Souweidane, Bander, Ramos, Thomson, Morgenstern. Reviewed submitted version of manuscript: all authors. Approved the final version of the manuscript on behalf of all authors: Souweidane. Statistical analysis: Souweidane, Bander, Ramos, Wembacher-Schroeder. Administrative/technical/material support: Souweidane. Study supervision: Souweidane.

\section{Correspondence}

Mark M. Souweidane: Cornell University, Weill Medical College, New York, NY.mmsouwei@med.cornell.edu. 